International Journal of Engineering \& Technology, $7(1.1)(2018) 477-481$
International Journal of Engineering \& Technology
SPC
Website: www.sciencepubco.com/index.php/IJET
Research paper

\title{
Energy Efficient and robust routing with RPAR Protocol in Ad Hoc Networks
}

\author{
Joshua Reginald Pullagura ${ }^{1 *}$, D. Venkata Rao ${ }^{2}$ \\ ${ }^{I}$ Vignan Foundation for Science, Technology and Research, Guntur \\ ${ }^{2}$ Narasaraopet Institute of Technology, Narasaraopet, India \\ *Corresponding author E-mail: pjreginald@gmail.com
}

\begin{abstract}
In ad hoc network, the topology of network changes frequently due to the mobile nature of nodes where the communication is possible without any fixed network infrastructure. Mobile nodes are battery operated and so energy efficient routing should be provided which increases the network life time. The existing routing mechanisms do not consider both hop count and energy of nodes for data transmission. In this paper we propose a routing mechanism where data transfer from source to destination is based on the minimum hop count and residual energy of mobile nodes. The proposed RPAR protocol shows better performance when compared to existing Energy power aware routing protocol .The analysis is carried out by using network simulator (NS-2), the simulation results shows that the proposed routing mechanism provides energy efficient and reliable routing in ad hoc networks.
\end{abstract}

Keywords: RPAR, EPAR, Residual energy, Energy efficient routing, Network life time.

\section{Introduction}

In ad hoc networks, the operations of nodes are based on the transmission range and battery power. Each node on the network involves in routing operations which provides the communication between different nodes. All nodes in the network can transmit their data to their intermediate nodes or to the destination node unless they have enough energy and the nodes must be within the transmission range. If the mobile node does not have enough energy it cannot transmit the data or participate in the network operation. So nodes energy consumption is very important factor to be considered for efficient data transmission. By using energy efficient routing mechanism in multi hop networks for data transfer the network life time can be increased. The extension of battery life time of node improves the nodal energy which increases the network performance.

The mobile nodes in ad hoc networks are self-managed and can work in remote areas without any maintenance or repair. Usually the data transfer from source to sink is carried out by using multiple hops with minimum energy consumption which reduces total expenditure of energy per packet transmission. The death of mobile nodes in the ad hoc networks will adversely affect services. Fig. 1 shows multi hop data transmission where the source will send route request to all the nodes participating in the network, when the request reaches the destination it will send route reply along with acknowledgement.

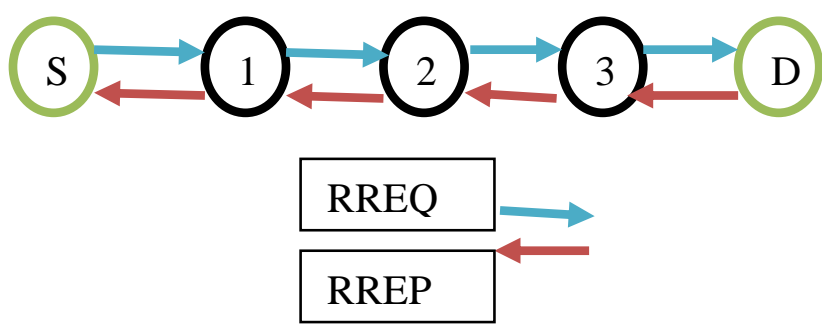

Fig.1: Multi hop Communication

The mobile nodes will dissipate energy when data is transmitted or received by the nodes. In multi hop transmission, the data from source to sink is reached through different intermediate nodes by considering the route request and route reply. For providing multi hop communication [3] from source to destination, the source will send the route request (RREQ) to the destination whether it will have free routes for data transmission. The destination will send a route reply (RREP) to the source which will have the information whether a free route is available or not. Based on the RREP the source will find a route with minimum hop count. The above multi hop communication only four hops are required for the data from source to reach destination. Here the source will send the data to node 1 - node2-node3 finally to the destination. Minimum hops from source to destination will decrease the time delay which improves the network performance. The mobile nodes in ad hoc networks can be function as both the data router and data originator [12], if some nodes does not have enough energy there may occur changes in topology which reorganize the network. Hence the data should be rerouted which causes more energy from other nodes and causes decrease in network life time .By 
considering the residual energy for data transfer, the network life time can be improved. The residual energy is the energy left out in a node after transmitting or receiving the packets. If this energy is less, the node may die out of energy and cannot participate in routing process. In order to avoid this problem nodes having maximum residual energy [1] is considered for data transfer that decreases the death of nodes in network so that the network life time can be improved. Thus, the work in this paper can be summarized as Calculate the number of packets which should reach the destination. After the route request and route reply consider an energy efficient path for data transfer based on hop count and residual energy. The remainder of this paper shows the related work in section 2, section 3 shows the Design and Implementation, Proposed routing procedure in section 4, Energy efficient data transmission is discussed in section 5 , section 6 shows proposed routing algorithm description, 7 shows performance evaluation and section 8 describes conclusion and future work.

\section{Related Work}

Routing plays a vital role in the transfer of packets from source to destination nodes. The protocols developed so far are either proactive or reactive. Few Hybrid Protocols are also developed. Hop Tree based Multicast routing also provides better results when compared to the traditional reactive and proactive protocols [2]. On demand protocols are usually source initiated protocols. Most of the previous work on routing in wireless ad-hoc Networks deals with the problem of finding and maintaining correct routes to the destination during mobility and changing topology. Node energy is one of the major constraints which decide the performance of ad hoc networks. If the node does not have sufficient energy for forwarding data, it cannot participate in the routing process. Usually the data transfer will be carried out based on the hop count from source to destination. So if a path has minimum hops it is used for the data transmission. But the continuous transmission of data through the same path decreases the node energy which degrades the network performances. Thus efficient energy consumption should be provided which increases the network life time so that an improvement in network performances can be obtained. In this section, some existing protocols are reviewed and the importance of energy efficient routing in ad hoc networks is over viewed.

Energy Power Aware Routing algorithm is an on demand source routing protocol that uses battery lifetime prediction [1]. The route discovery process is initiated by the Source node. In the network node power consumption is mainly due to transmission or reception of packets. The intermediate nodes in the network also lose their power as they need to forward the packets based on the destination nodes location. In the energy power routing protocol the path is chosen based on energy or battery power of the node. First, the battery power for each path is computed. The path is then selected by considering the path with the maximum lowest hop energy.

\section{Design and Implementation}

In longer paths, usually the energy of intermediate node drains when more number of packets are sent through same path or when any intermediate node in that path becomes malicious. In the proposed algorithm the amount of consumed energy for selected route is given as

$$
R E=\sum_{i=1}^{N} E_{N}
$$

Where $\mathrm{N}=$ No. of nodes involved in packet transmission

$\mathbf{E}=\begin{aligned} & \text { Energy } \\ & \text { consumed to } \\ & \text { transmit a } \\ & \text { packet }\end{aligned} \quad \begin{aligned} & \text { Energy } \\ & \text { consumed to } \\ & \text { receive a } \\ & \text { packet }\end{aligned}$

$\mathbf{E}=\mathbf{T}_{\mathbf{x}} \cdot \mathbf{t}+\mathbf{R}_{\mathbf{x}} \cdot \mathbf{t}$

$$
\begin{aligned}
& \mathrm{T}_{\mathrm{x}}=\text { Transmission power } \\
& \mathrm{R}_{\mathrm{x}}=\text { Reception power } \\
& \mathrm{t}=\text { time }
\end{aligned}
$$

The energy required for one packet transmission is taken as 1units and packet size is considered as 512 bytes. The ad hoc networks mainly consist of different mobile nodes, a set of base station and trusted party .The nodes are distinguished by their mobility, processing time, energy consumption and memory capacity. All nodes in ad hoc networks have limited battery power, so node energy consumption is very essential [6] .Hence power efficient energy aware routing protocol is implemented. Here the data transmission from source to sink is carried out depending on the minimum hop count and the residual energy of each node. When the energy level of the node decreases to certain level it will intimate the source and source will find an alternate path for data transmission. Hence by providing an energy efficient routing, life time of the network can be improved

\section{Proposed Routing Procedure}

In Proposed Mechanism the route is selected based on battery power of nodes and routing mechanism is on demand Source initiative which utilizes the principle of minimum hop mechanism. As the energy of node drains in selected path, the change of path for data transfer is carried out, if the energy level of node decreases to certain level, life time of the network will reduce with more number of transmissions. The data transmission from source to sink node is carried out by considering the residual energy and minimum hop count .The node having maximum residual energy [1] is considered for data transfer .High throughput is always desired for any routing mechanism[5]

Fig. 2 shows an example of proposed routing mechanism carried out in a network. Here the data from source node to sink node can be transmitted through two different routing. The first route (S-34-5-D) has three nodes with residual energy 2, 3, 6 respectively and second route (S-1-2-D) has two nodes having residual energy 5 and 3 respectively. From these two routes, second routing mechanism is preferred for data transfer from source to sink node because it has minimum hop count with maximum residual node. Even though, first route will have the highest residual energy it is not considered for data transfer since the hop count for this route is high compared to other one which increases the time delay and adversely affect the network performance. Hence the proposed routing mechanism considers both the hop count and the residual energy of the node for data transfer thereby improving network life time 


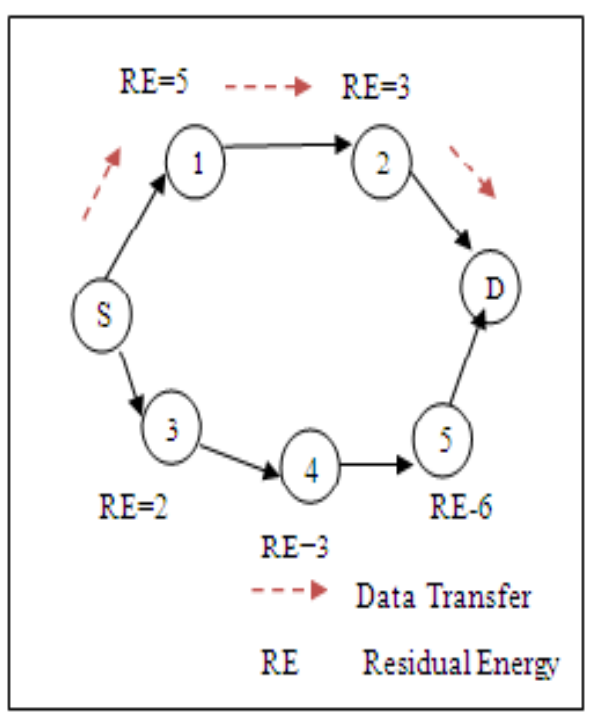

Fig. 2: Proposed routing procedure

\section{Energy Efficient Data Transmission}

Energy is one of the important parameter which decides the performance of ad hoc network [7]. The mobile nodes may run out of battery if same nodes are used for the data transfer for longer periods. Usually during route establishment from the source to destination Minimum hop count is considered and the node energy is neglected. In order to improve the network performance, energy efficient routing is considered [12] where the data transfer from source to destination will depend on the residual energy of the node and minimum hop count. Residual Energy of a node depends upon the amount of data transmitted or received by the node which can be calculated as,

Residual Energy=Initial Energy of a node- remaining energy of node after transmitting the data at time $t$.

Initially all the nodes participating in the network will have equal energy, after transmitting the data through the nodes, node energy decreases which reduces the network life time. So in order to improve the network life time [8],[10], the nodes participating in the network will inform the source to change the path, if its residual energy is less than a threshold value. The routing protocol should function effectively under different network conditions and which should consumes minimum node energy consumption. Power efficient energy aware routing protocol [3] is implemented for providing the data transmission based on the residual energy and minimum hop count. Cluster based approach also reduces energy a little bit [14] .The function of the protocol comprises of route discovery, routing table and route maintenance.

a) Route Discovery

Different routes from source to destination can be obtained by sending a route request (RREQ) by Source Node to all nodes participating in the network. When RREQ reaches the destination, it will send route reply (RREP) back to the source. Here the nodes with minimum hop count and having highest residual energy [4] is considered for data transfer from source node to sink node.

b) Routing Table

Routing Table [11] will contain all the details of the mobile nodes, its neighboring nodes, hop count, residual energy. Based on the values on the routing table efficient mobile nodes are considered for data transfer. If the residual energy of the nodes decreases to a certain level it will intimate the source to find an alternate path for data transfer. Depending up on the values in the routing table, the source will find an alternate path for data transmission from source to destination.

c) Route Maintenance

The route established from source to destination should be maintained under different environmental conditions. The route is maintained by sending the route error (RERR) messages. While data transferring if any problem occurs, the intermediate node will send RERR messages to the source so that it can use an alternate path for data transmission

\section{Proposed Routing Algorithm}

The proposed routing protocol considers different modules for providing efficient data transmission and improving the network performances. Different modules are

a) Calculate number of packets

Numbers of packets are calculated, based on the transmission and retransmissions of packets and energy of each node is evaluated.

b) Insert the values to routing list

After initialization of the list, various information about nodes and paths like source id, destination id, packet number, sequence number, hop count, residual energy are stored.

c) Path Selection

Whenever a node tries to send a data, it initially sends route request messages which contain packet type, source id, destination id, packet number. Based on this information, the intermediate node will check the destination id if it matches it will check about the source information. If it is already available it will check which is the better path based on the hop count and residual energy, if a new path is found better it will generate RREP and send the data through that path otherwise it will stick to the old one. If the destination id is not matched it will store that value in the list and forward it to neighbors for next process.

d) Residual Routing

When intermediate node gets troubled by loosing energy, it will send request to the source, then the source will consider available alternate path for data transfer. If the nodes are not troubled, source will automatically select an alternate path when node energy reaches threshold level.

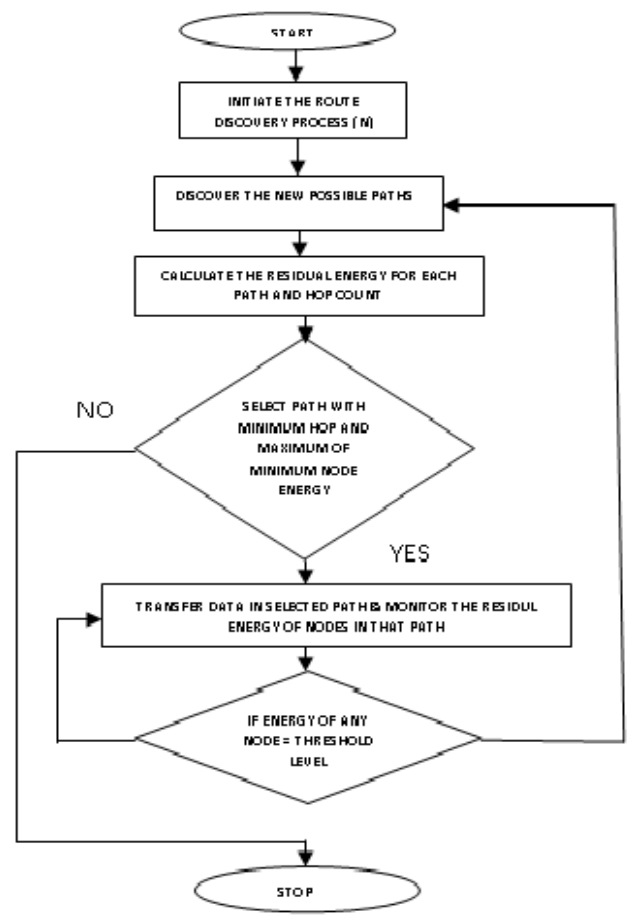

Fig. 3: Flow diagram 


\section{Performance Evaluation}

The performances of energy efficient routing based on node residual energy are simulated by using network simulator 2.35 . Results can be obtained by in the form of trace analysis, Network animator (NAM) window and XY graph. Trace analysis shows the packet trace on individual link. The data transmissions from source to destination through non traceable nodes are analyzed by using NAM window. Fig. 4 shows the output in NAM window where the data transmission from source to destination nodes are based on the residual energy of nodes

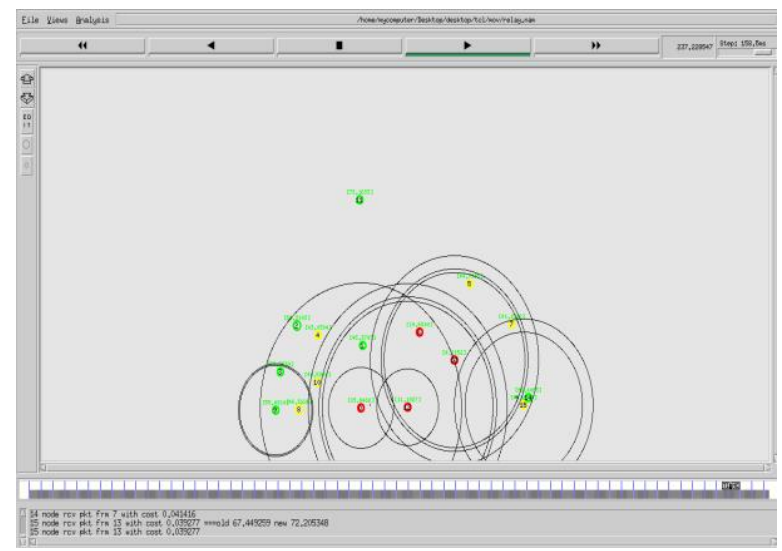

Fig. 4: NAM Window showing data transmission between intermediate nodes

Table 1: Simulation Parameters.

\begin{tabular}{|c|c|}
\hline Number of nodes & 120 \\
\hline Area & $20000 \times 20000$ \\
\hline Packet size & 512 bytes \\
\hline Traffic type & CBR \\
\hline Routing Protocols & RPAR,EPAR \\
\hline
\end{tabular}

Performance metrics can be used to evaluate the network performance by considering different parameters. Delay and Energy consumption are the two important metrics considered here

\subsection{Delay}

Delay is the time taken for the data to reach the destination. Delay should be short for improving the network performance so that the data can reach the destination with minimum duration. Fig. 5 shows that delay for the proposed mechanism and is shorter because the route with minimum hop count is selected. If the intermediate nodes have any problem they will find an available alternate path for data transfer which decreases the delay during packet transmission

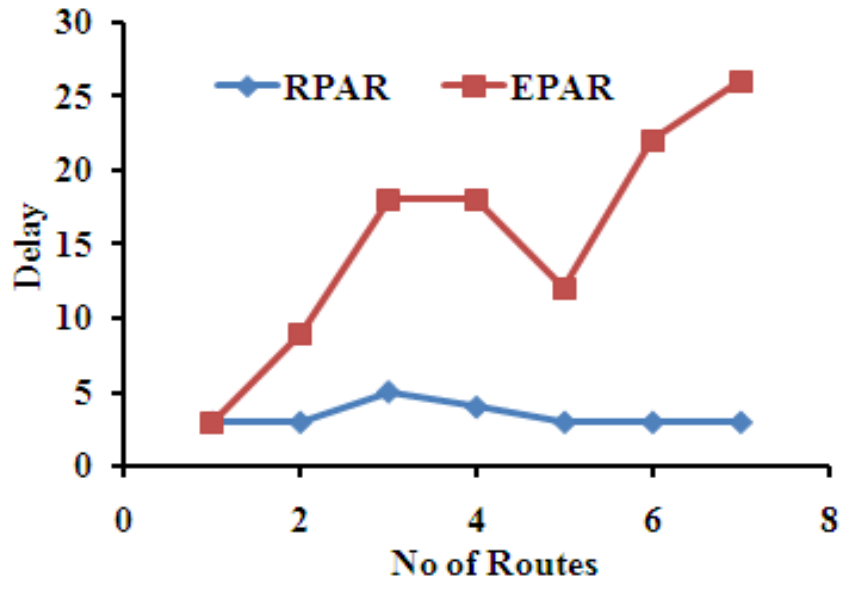

Fig.5: Delay comparison of RPAR and EPAR for different number of routes

\subsection{Energy Consumption and Life Time}

Energy consumption of a node is the total energy consumed by a node to transfer the data.. If energy consumption is less the life time of the route will be higher[6]. In order to provide energy efficient routing, the node should use minimum energy for transferring the data to the destination[9]. Fig.6 shows the comparison of Number of hops used in transmission of first 10 cycles. Energy consumption and network performances are inversely related to each other. The energy consumed by the proposed RPAR is uniformly dissipated and is less when compared to conventional EPAR routing mechanism due to minimum number of hops used for data transfer

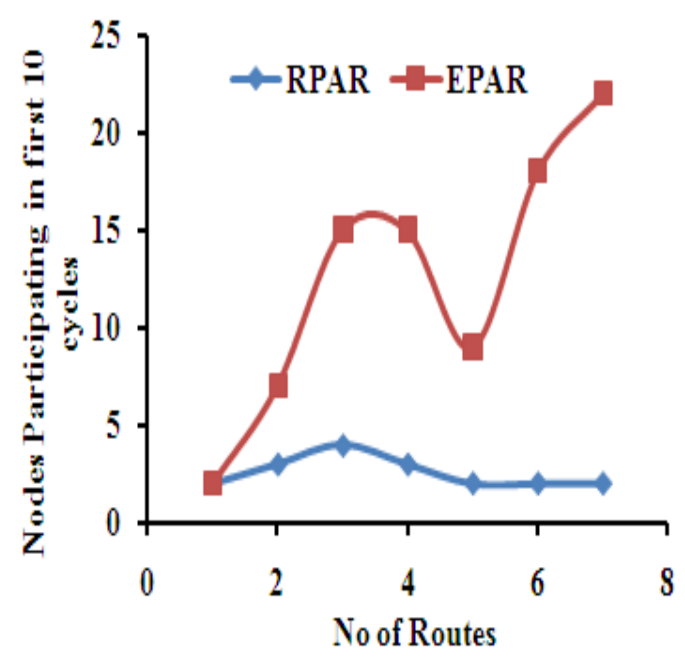

Fig. 6: Nodes participating in first 10 cycles

The proposed routing mechanism will consider node energy for data transfer. If the node energy reduces to a certain Threshold level, an alternate path is used for data transfer. The alternate path will improve the network life time because residual energy based data transmission decreases the death of nodes in the network. Here we tried to balance battery energy by switching the routes. Fig 7 shows less no of nodes are used for data transfer in RPAR where as EPAR shows constant increase of nodes participating in data transfer as number of routes increases. Here the threshold energy level is fixed to 5 units.

Life Time is one of the important parameter. It is the time that node is operative. Even though there are many nodes with many routes, Life Time depends upon the energy that a single node has. 
In any route if energy of any node is low than life time of that route will be limited. Depending upon lowest energy node in our scenario both RPAR and EPAR protocols exhibit same life time. Fig.8. shows the Life Time plot for the two Protocols

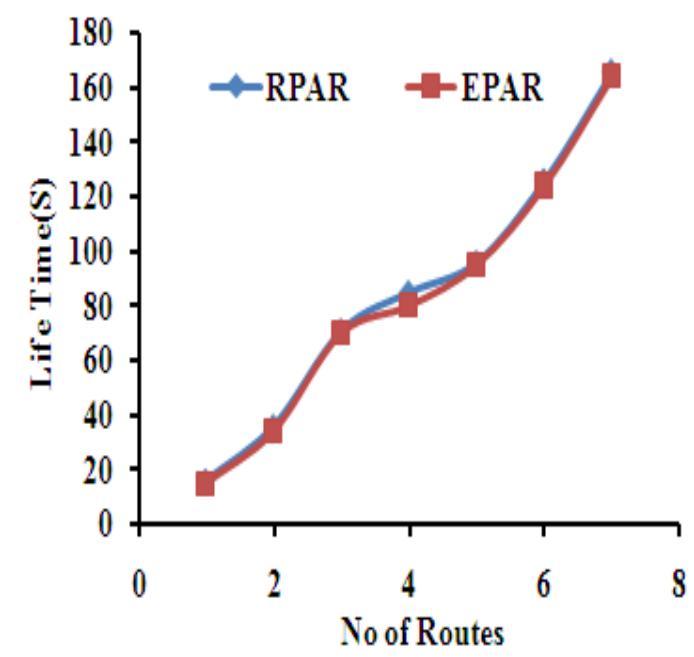

Fig. 7: Life Time for both existing and proposed routing mechanisms

\section{Conclusion and future scope}

Proposed routing mechanism uses both the residual energy and minimum hop count for data transmission. Node having maximum residual energy and minimum hop count route was considered for path selection. Energy is monitored periodically hence Residual energy based routing decreases the death of nodes in the network which improves the network life time. Number of nodes that consume energy is also low in the proposed algorithm and it has shorter delay and more life time when compared to the EPAR protocol and the communication will provide high quality of services by achieving minimum delay. Thus the problem of energy consumption can be reduced by this low cost method. A node some time exhibit malicious nature due to various attacks [13] .Vampire attack is one of the deadly attacks that drain the nodes power. This work can be further extended to the network under various attacks to check the robustness of the algorithm.

\section{References}

[1] Shivashankar, Hosahalli, Narayanagowda Suresh, Golla Varaprasad and Guruswamy jayanthi "Designing Energy Routing Protocol with Power Consumption Optimization in Manet"IEEE Transactions on emerging topics in computing VOL 2 , NO 2, June 2014.

[2] Joshua Reginald Pullagura, Dr D.Venkata rao " An efficient and reliable cooperative Multicast routing based on Hop tree in $\mathrm{Ad}-$ hoc Networks" IEEE-ICACCS-2016; DOI: 10.1109 /ICACCS. 2016.7586365.

[3] J.Vazifehdan,R.Venkatesha Prasad and I.Niemegeers, "EnergyEfficient Reliable Routing Considering Residual Energy in Wireles Ad hoc Networks",IEEE Trans, on Mobile Computing,Vol.13,No.2,February 2014.

[4] Q.Dong,S.Banerjee,M.Adler,and Misra, "Minimum Energy Reliable Paths Using Unreliable Wireless Links",Proc. ACM MobiHoc,pp.449-459,May 2005.

[5] D.S.J.De Couto,D.Aguayo,J.Bicket,and R.Morris,"A High Throughput Path Metric for Multi-Hop Wireless Routing",Proc.ACM MobiCom,pp.134-146,2003 .

[6] S.Singh and C.Raghavendra"PAMAS-Power Aware Multi Access Protocol with Signalling for Ad Hoc Neworks,"ACM Computer Comm.Rev.Vol.28,pp.5-26,1999.

[7] J.Gomez,A .T.Campbell ,M.Naghshinneh and C.Bisdikian,"PARO: Supporting Dynamic Power Controlled Routing in Wireless Ad Hoc Networks",Vol.9,No.5,pp.443460,2003 .
[8] C.Toh,"Maximum Battery Life Routing to Support Ubiquitous Mobile Computing in Wireless Ad Hoc Networks",IEEE Comm. Magazine,Vol.39,No.6,pp.138-147,June 2001.

[9] D.Kim,J.J.G.Luna Aceves,K.Obraczka,J.Carlos Cano, and P.Manzoni,"Routing Mechanism for Mobile Ad Hoc Networks Based on the Energy Drain Rate",IEEE Trans.Mobile Computing, Vol.2,pp.161*173,April-June 2003.

[10] J.H.Chang and L.Tassiulas,"Maximum Life Time Routing in Wireless Sensor Networks", IEEE/ACM Trans.Networking, Vol.12, No.4,pp.609-619,August 2004.

[11] D.J.Vergados,N.A.Pantazis,and D.D.Vergados, "EnergyEfficient Route Selection Strategies for Wireless Sensor Networks",Mobile Networks and Applications,Vol.13,No.34,pp..285-296, August 2008.

[12] A,Misra and S.Banerjee,"MRPC:Maximizing Network Lifetime for Reliable Routing in Wireless Environments",Proc.IEEE Wireless Comm.and Networking Conf. (WCNC'02), pp.800806,2002 .

[13] Joshua Reginald Pullagura, Srikant Perala, Dr.D.venkata rao "Attacks on Mobile Adhoc Networks:A Survey. IJCA Proceedings on Emerging trends in Computing 2017

[14] A.Akhtar, A. Ali Minhas, and S. Jabbar, "Energy Aware Intra Cluster Routing for Wireless Ad Hoc Networks", International Journal of Hybrid Information Technology Vol.3,No.1, January, 2010. 